\title{
Clinical Measures of Disease Progression in Amyotrophic Lateral Sclerosis
}

\author{
Seward B. Rutkove
}

Published online: 13 January 2015

(C) The American Society for Experimental NeuroTherapeutics, Inc. 2015

\begin{abstract}
Progressive weakness remains the clinical hallmark of amyotrophic lateral sclerosis (ALS). Accordingly, a variety of tools has been developed to capture this disease feature, including questionnaires, such as the ALS-functional rating scale, strength testing, pulmonary function tests, electrophysiologic measures, including motor unit number estimation, and imaging techniques. Despite this plethora of approaches, there is little agreement as to what measures to use in a given clinical trial or in the clinic during routine patient care. Part of the reason for this uncertainty is that ALS is a remarkably protean disease. Some individuals progress rapidly, others slowly; some patients have considerable upper motor neuron dysfunction, whereas others have little; and there is considerable variation in the sequence of body regions affected, in some the disease beginning in the bulbar musculature and in others in one arm or one leg. Here, I present a variety of basic and more complex clinical measures for potential use in therapeutic trials with the aim of offering a balanced and practical set of recommendations, as well as considerations for future studies.
\end{abstract}

Key Words Amyotrophic lateral sclerosis $\cdot$ functional measures $\cdot$ biomarkers $\cdot$ electrophysiology

\section{Introduction}

At first consideration, measuring the progression of amyotrophic lateral sclerosis (ALS) appears to be a straightforward problem. In contrast to nearly all other degenerative neurological and neuromuscular conditions, the progression is so rapid that

\footnotetext{
S. B. Rutkove $(\bowtie)$

Beth Israel Deaconess Medical Center, Harvard Medical School, 330

Brookline Avenue, Boston, MA 02215, USA

e-mail: srutkove@bidmc.harvard.edu
}

patients or caregivers can often describe progressive loss of capabilities from month to month or even week to week. In contrast, other diseases, such as inclusion body myositis or adult forms of muscular dystrophy, progress so slowly that patients have to compare carefully their functioning from year to year or decade to decade to determine whether they have actually worsened. Moreover, as ALS eventually affects all motor systems throughout the body, there are many potential regions and techniques that can be applied, from simple dynamometry, to assess appendicular function, to pulmonary function tests, to assess the muscle of respiration. One would think that with this abundance of measures, there should be little challenge to identifying sensitive measures of deterioration.

Yet despite its speed of progression and the plethora of potential assessment tools at our disposal, ALS presents unique challenges. In fact, choosing which tests to utilize when testing therapeutics in a clinical trial is not a simple matter. Most of these challenges relate to the disease's remarkably protean nature: 2 people with the same disease can have extremely different presentation and courses. Such heterogeneity adds a challenge that is not present for many other neuromuscular conditions.

The first and most obvious challenge in ALS is that the body regions affected by the disease vary from patient to patient. Clearly, someone with primary bulbar symptoms early in the course may require a different outcome measure than someone with predominantly lower extremity limb weakness. This variability creates a therapeutic testing challenge as during the 6-12 months of a typical clinical trial, 2 people could have very different areas deteriorating.

A second challenge is the variation in upper versus lower motor neuron involvement in different people. Certain tests may be better geared toward evaluating lower rather than upper motor neuron involvement, such as motor unit number estimation. In contrast, progressive dysarthria due to upper motor neuron loss, may be challenging to quantify. 
A third clear challenge is the remarkable variability in the rates of decline across patients. Some patietns with ALS may live less than a year from the onset of symptoms, whereas others will live for more than a decade. This variability proves to be an especially challenging component in clinical therapeutic trial design and in determining the most appropriate outcome measures to employ.

In this review, I will work my way through from the most "subjective" measures (clinical questionnaires) to functional measures, electrophysiological tools, and, finally, muscle imaging (central nervous system imaging is discussed elsewhere in this special issue). A summary is also provided in Table 1 .

\section{Rating Scales and the ALS Functional Rating Scale-Revised}

Several early efforts at developing a functional rating scale for clinical trial use were pursued in the 1970s and 1980s, including a scale described by Norris, first used in a study of guanidine (1974) [1], and then, a decade later, the Appel Scale (1984) [2]. However, both of these early scales were complex and time consuming to administer. Thus, in the 1990s the most well-known and utilized measure of disease progression, the ALS functional rating scale (ALSFRS) was developed [3]. The scale was based on responses to 10 questions, each graded on a scale of 0 to 4 , in all the different domains that might be affected by the disease, including bulbar function, respiratory function, and upper and lower limb function. The original ALSFRS was replaced with the ALSFRS-revised (ALSFRSR) just a few years later when it was recognized that additional measures of respiratory decline were needed [4]. The new scale consists of 12 questions that total 48 points.

The validity of the scale has been shown in several studies to be high, in its being relatively sensitive to disease progression and being reliable [5-7]. The rate of deterioration also correlates with survival [8]. An obvious advantage of such a measure is that it is very quick to perform and can even be completed over the telephone or online, making it simple to obtain regular data, even

Table 1 Summary of measures to evaluate progression of amyotrophic lateral sclerosis

\begin{tabular}{|c|c|c|c|}
\hline Category & Specific method & Advantages & Disadvantages \\
\hline \multirow[t]{4}{*}{ Clinical measures } & ALSFRS-R & $\begin{array}{l}\text { - Easy to employ } \\
\text { - Has been shown to correlate to survival and is } \\
\text { robust in several trials }\end{array}$ & $\begin{array}{l}\text { - Relatively insensitive to progression over } \\
\text { short periods of time } \\
\text { - Affected by mood/effort }\end{array}$ \\
\hline & Strength testing & $\begin{array}{l}\text { - Universally available } \\
\text { - Can be used to evaluate limbs effectively }\end{array}$ & $\begin{array}{l}\text { - Affected by mood/ effort } \\
\text { - Reliability uncertain } \\
\text { - Insensitive early and late in disease } \\
\text { - Bulbar testing less clear }\end{array}$ \\
\hline & FVC & $\begin{array}{l}\text { - Repeatable and quantifies the health of the most } \\
\text { critical activity for sustaining life }\end{array}$ & $\begin{array}{l}\text { - Only evaluates } 1 \text { region } \\
\text { - May not show changes early in disease } \\
\text { - Later in disease it may be affected by bulbar } \\
\text { weakness and poor lip closure }\end{array}$ \\
\hline & $\begin{array}{l}\text { Bulbar-specific } \\
\text { measures }\end{array}$ & - Easy to perform & - Not as well studied as longitudinal metrics \\
\hline \multirow[t]{4}{*}{ Electrophysiological } & CMAP/NI & $\begin{array}{l}\text { - Easy to obtain } \\
\text { - May be very sensitive to decline }\end{array}$ & $\begin{array}{l}\text { - Has not been employed extensively } \\
\text { - Limited to distal muscles }\end{array}$ \\
\hline & MUNE & $\begin{array}{l}\text { - Provides approximation of actual motor neuron } \\
\text { number and rate of actual motor neuron loss }\end{array}$ & $\begin{array}{l}\text { - Technically challenging } \\
\text { - Potentially insensitive early in disease } \\
\text { - Limited to distal muscles }\end{array}$ \\
\hline & EIM & $\begin{array}{l}\text { - Easy to utilize } \\
\text { - Can assess many muscles }\end{array}$ & $\begin{array}{l}\text { - Mechanisms of change over time not intuitive } \\
\text { - Limited studies evaluating its potential at this time }\end{array}$ \\
\hline & Excitability testing & $\begin{array}{l}\text { - Potentially useful for early diagnosis } \\
\text { - Some measures correlate with survival }\end{array}$ & $\begin{array}{l}\text { - Limited data on progression } \\
\text { - Requires specialized equipment } \\
\text { - May be challenging to use in multicentered studies }\end{array}$ \\
\hline $\begin{array}{l}\text { Muscle imaging } \\
\text { (MRI and US) }\end{array}$ & & $\begin{array}{l}\text { - Relatively easy to use, (especially for US) } \\
\text { - Conceptually straightforward }\end{array}$ & $\begin{array}{l}\text { - Studies have suggested that they are insensitive } \\
\text { to change }\end{array}$ \\
\hline Serum and CSF markers & & $\begin{array}{l}\text { - If biomarkers can be identified in serum, high } \\
\text { ease of use } \\
\text { - Correlate to rate of progression }\end{array}$ & $\begin{array}{l}\text { - None currently effective at showing } \\
\text { longitudinal change }\end{array}$ \\
\hline Survival & & - The ultimate outcome of clinical value & $\begin{array}{l}\text { - Not really a biomarker, but rather an endpoint } \\
\text { - Can be impacted by factors unrelated to the rate } \\
\text { of disease progression }\end{array}$ \\
\hline
\end{tabular}

ALSFRS-R = Amyotrophic Lateral Sclerosis Functional Rating Scale-Revised; FVC $=$ forced vital capacity; CMAP/NI = compound motor action potential/neurophysiological index; MUNE = motor unit number estimate; $\mathrm{EIM}=$ electrical impedance myography; $\mathrm{MRI}=$ magnetic resonance imaging; $\mathrm{US}=$ ultrasound; $\mathrm{CSF}=$ cerebrospinal fluid 
if the patient is unable to return to the clinic for evaluation [5]. It also ensures that all realms of potential disease progression are being captured. On average, patients lose about 1 point per month as the disease progresses $[9,10]$; thus, it offers a relatively simple and easily applied measure of progression. The US Food and Drug Administration (FDA) also approved incorporation of the questionnaire as an outcome measure (in conjunction with survival via joint-rank study design) in a large trial of dexpramipexole in ALS [10]. Thus, the scale's value has recently taken on added significance.

Despite its clear benefits, the ALSFRS-R has a number of limitations, which should be highlighted. First, it is not especially sensitive to disease progression, and in those patients who are progressing relatively slowly, capturing any change may require several months. Accordingly, phase 3 clinical trials, such as the recent dexpramipexole study [10], may need to be relatively long (i.e., 1 year) in order to identify significant disease progression. Second, it is by its nature a subjective test and thus its has the potential to be affected by mood, potential unblinding, and therapeutic optimism [11]. For example, patients in a drug study may report less progression than those not on an experimental drug simply because they believe the drug might be working. Indeed, a natural history study evaluating ALSFRS-R along with other measures actually showed the ASLFRS-R to be more sensitive to progression than had been reported in other studies [12], perhaps because there was no subjective bias toward a drug having a therapeutic effect. Another limitation of the ALSFRS-R is that it may be affected by the degree of social support and the presence of depression [13]. Finally, a recent Rasch analysis found that the test failed rigorous measurement standards, with the authors of this analysis calling for its revision [14].

The ALSFRS-R is not the only questionnaire or scale in use to evaluate disease status in the ALS. Self-reported outcomes have been studied, including a recent investigation that successfully used the "patientslikeme" website (www. patientslikeme.com) to prove lithium's ineffectiveness in the disease [15]. However, such an approach could not substitute for a placebo-controlled study to achieve FDA approval. Similarly, quality-of-life measures could also be valuable [16, 17], but have not garnered general acceptance as a clinical trials metric. The undeniable value of such scales and questionnaires is that they are incredibly cost-effective, can be done from a distance, can be comprehensive in their assessments, and can capture an element of personal well-being not possible with all of the other approaches to be discussed next.

Strength Measurements, Including Handheld Dynamometry, Maximal Voluntary Isometric Contraction Testing, and Manual Muscle Testing

As weakness is the major clinical feature of ALS, the quantification of strength in a patient over time would appear to be a robust and sensitive approach for assessing disease progression. The most obvious and basic measure is standard manual muscle testing [18], with grading of muscle strength in each individual limb on a 0-5-point Medical Research Council scale [19]. Whereas this approach works, is generally considered reliable $[20,21]$, and was used for a number of ALS studies, even as recently as 2007 [22], it has mostly fallen out of favor. This is true because of the technique's inability to detect subtle degrees of weakness and because it is affected by examiner strength [23]. For these reasons, approaches for improving the quantification of weakness have represented a longstanding effort.

The first major innovation in this regard was the development of the Tufts Quantitative Neurological Examination. This test basically consisted of a measure of maximal voluntary isometric contraction testing using an electronic stain gauge in conjunction with pulmonary and bulbar measures [24]. The isometric strength aspect of this work was further improved by the creation of a "megascore," or a z-averaged score across a predefined set of muscles [25]. Such maximal isometric voluntary contraction was incorporated into a variety of trials in the 1990s and early 2000s [9, 26-28]. While providing good data, the methodology was very challenging to institute, as it required a large plinth upon which the debilitated patient needed to be placed; this became especially challenging as patients became too weak to transfer. Moreover, testing required highly trained personnel not only in using the apparatus effectively, but also in ensuring that the patient was not moving during the procedure. It was also limited to a relatively small set of muscles and one study even suggested that it performed no better than manual muscle testing [29]. Given that the costs of the systems were relatively high (approximately $\$ 10,000$ ) and the entire system quite bulky, their use fell out of favor in most clinical trials by the mid-2000s.

A newer approach to performing such isometric testing has recently been introduced using the ATLIS system [30]. Based on similar principles, it is also a form of isometric contraction testing, but one that is considerably easier to employ as the system is smaller and the outcomes easier to obtain. Several upper and lower extremity muscle groups can be measured using this approach and a normalized score (akin to those used in pulmonary function testing) is obtained for easier data interpretation [31]. This promising effort continues to be under study at this time.

Handheld dynamometry (also called quantitative myometry) is now probably the most commonly used methodology for assessment of muscle strength in clinical trials. Its use was first suggested as an alternative to the more complex maximal isometric contraction systems in 1999 [32]. In handheld dynamometry, the examiner holds a small-strain gauge that the patient pushes against using a variety of muscle groups, and a force measurement is obtained. The concept dates back, remarkably, to 1763 , when the The Graham- 
Desaguliers force dynamometer was first introduced [33]. The test is usually repeated 2-3 times to ensure consistency of the data for a given muscle; then the examiner moves on to the next muscle. This approach is appealing as it requires no bulky equipment, relatively limited training (mostly geared toward ensuring the patient is positioned appropriately for each type of contraction being performed), and can be performed on a variety of muscle groups. However, a major limitation of handheld dynamometry, which also holds true with simple manual muscle testing, is that it is challenging to measure people who are relatively strong, as it is difficult to create sufficient force to overcome their contraction (a necessity to achieve good quality data). Indeed, the testing can become quite uncomfortable in patients who are not clinically weak in a given muscle group; the end result will be that the examiner's own strength rather than the patient's is being measured, and the data may be entirely unreliable [34]. In patients who are very weak reliability also may be challenging [35]. Thus, in a given patient, there is only a limited range over which the technique is reliable - namely those muscles that are mildlyto-moderately weak. And as even in the best of circumstances there is considerable noise in each measurement, it does require that multiple muscles be measured and averaged to ensure consistent data. Despite these limitations, given its ease of performance, handheld dynamometry is likely to still be the preferred method of strength examination in clinical trials for the foreseeable future.

All forms of strength testing have some limitations worth considering. First, they are effort and mood dependent, and a depressed patient may have difficulty in performing at his/her peak performance. Moreover, a patient with any cognitive issues, including frontotemporal dementia, may not be able to follow commands effectively. Second, the metrics cannot evaluate bulbar or other cranial nerve weakness effectively. Third, increased tone associated with upper motor neuron degeneration may make it difficult to obtain accurate strength measurements. Finally, as ALS often progresses most conspicuously in a subset of limbs in a given patient during a clinical trial, the data from the nonprogressing limbs may only add noise to the measurement. Performing strength testing on just a subset of muscles in just 1 or 2 limbs may be an approach to correcting this issue, but has not been studied.

\section{Respiratory Function}

As respiratory dysfunction remains the usual cause of death in ALS, measurement and quantification of respiratory function in these patients is, on the face of it, one of the most important measures to obtain $[36,37]$. As respiratory dysfunction is mainly caused by muscle weakness and produces a restrictive pattern, one of the most utilized measures in clinical trials is the forced vital capacity (FVC) [22, 27, 38]. FVC essentially measures the full extent of a breath capable by a patient, excluding the dead space of the lung and upper airways. Other measures of pulmonary function in ALS have also been studied, including maximal voluntary inspiratory or expiratory pressures, but it is not clear that these offer any advantage over the FVC [39].

A major challenge to all forms of pulmonary testing is that the test becomes increasingly unreliable if there is bulbar weakness and the patient cannot achieve a good mouth seal around the device. In addition, FVC testing is generally unhelpful in patients with early disease as the values may be relatively insensitive to change early on.

\section{Measures of Bulbar Dysfunction}

Clinical measures of bulbar dysfunction represent a separate category of testing procedures. But as much of the effort in characterizing disease progression in ALS is focused on progressive weakness of the limbs, the bulbar-specific measures continue to be relatively limited in number. First, the ALSFRS-R bulbar subscore has been utilized as a simple test [4], but only contains 2 items, so is fairly limited in its granularity. In lieu of this rough assessment, a battery of tasks has been described as useful in assessing ALS [40, 41], including the Iowa Oral Performance Instrument measurements of tongue, lip, and cheek strength [42]. The Sydney Swallow Questionnaire may also be useful in this regard [43]. Other approaches, including magnetic resonance imaging (MRI), electrical impedance myography, and quantification of standard swallowing studies are also being explored as potential markers of disease status, but will not be discussed here further.

\section{Peripheral and Central Electrophysiological Biomarkers}

As nerve conduction studies and needle electromyography remain the main methods for confirming a diagnosis of ALS, it is perhaps not surprising that electrophysiologic methods also provide excellent approaches for monitoring clinical disease progression. However, needle electromyography, which is so critical in helping establish the diagnosis of ALS, is not very useful in identifying disease progression. This is because the technique is generally employed in a subjective fashion and quantitative methods are timeconsuming to perform and require considerable expertise [44].

\section{Compound Motor Action Potential and the Neurophysiological Index}

The compound motor action potential (CMAP) is generated by the near-simultaneous depolarization of all muscle fibers in a muscle (or group of muscles) via supramaximal stimulation of a single nerve [45]. The CMAP is a standard fixture of nerve conduction studies, with reductions in amplitude 
usually corresponding to motor axon-loss neuronal pathology. Despite this, most ALS studies have not evaluated the use of the CMAP as a measure of disease progression; rather, they have focused on motor unit number estimate (MUNE). However, there are considerable CMAP data as a CMAP is needed in order to calculate the MUNE. And, in fact, the median and ulnar CMAPs actually show substantial decline over time in most patients with ALS, as demonstrated by 2 recent MUNEfocused studies [46, 47]. The biggest challenge to the CMAP is its reliability, which is sensitively dependent on electrode positioning, limb and hand positioning, and temperature. Not surprisingly several studies that have looked at CMAP reliability have shown mixed results $[48,49]$. However, by carefully trying to maximize the CMAP value and being consistent about electrode placement and limb position, it may be possible to more effectively use the CMAP as a primary marker of disease progression.

Another approach that incorporates the CMAP was first suggested by Swash and de Carvalho in 2004 [50]. Termed the "neurophysiological index" (NI), it is defined as $\mathrm{NI}=($ ulnar CMAP amplitude/distal motor latency) $\times \mathrm{F}$ wave persistence [50]. By dividing the CMAP by the distal motor latency and then multiplying by the $\mathrm{F}$ wave persistence (the former gradually increasing in progressive disease and the latter gradually decreasing), the premise was that the NI would be a sensitive marker of disease progression. As these data are easily obtainable with a standard nerve conduction system and only require a few more stimuli beyond a typical nerve conduction study, it is easy to apply. Unfortunately, there has been relatively little follow-up research of this suggested biomarker. However, a recent study has shown that it is very sensitive to disease progression over a several-month period [51], but it also showed its sensitivity was mainly due to the CMAP amplitude rather than the F wave persistence or the distal motor latency. In addition, there has been no longer-term study and its test-retest repeatability is unknown. Nonetheless, I believe that further, more carefully performed studies of both CMAP and NI are warranted.

\section{MUNE}

MUNE remains one of the most theoretically compelling electrophysiologic measures of disease status in ALS. In MUNE, an attempt is made to estimate the number of motor neurons innervating a muscle or muscle group [52]. This is achieved by first obtaining a CMAP using standard supramaximal nerve stimulation usually from the median or ulnar nerves. Then, using one of several techniques, an effort is made to obtain the average size of the single motor unit potentials (SMUPs) that contribute to the CMAP. The most obvious and first-described approach is by incremental stimulation [52], in which the stimulation intensity over the nerve is gradually increased from subthreshold values and the size of the steps in amplitude with successive elevations in stimulation is noted. The average size of a step is then measured and that size divided into the CMAP to obtain the MUNE. Another approach is what has been called the multipoint stimulation technique in which the nerve is stimulated at different points along the length of the nerve at very low levels with an effort to obtain different low threshold SMUPs and measuring the individual amplitude of each [53]. After collecting data and averaging 10 such SMUPs, the values are divided into the CMAP to obtain the MUNE. Other methods incorporate various versions of these basic approaches, some incorporating the use of $\mathrm{F}$ waves or needle recording of the action potential [54]. One other, more recent, version combines both multipoint stimulation and incremental techniques [46]. Another variation, termed MUNIX, has been developed in which, after obtaining the CMAP, contraction of the muscle is made at various intensity levels and the surface interference pattern measured and divided into the CMAP value [55].

While MUNE is conceptually appealing, it is challenging to implement as it requires considerable training and can be time consuming to perform well, with a great deal of real-time decision-making (although this remains less of an issue with MUNIX). With practice it does become fairly easy to perform, but to the uninitiated the procedure can seem daunting. While studies have suggested good repeatability across a group of individuals [56], test-retest variation for individual data can be higher, with variation of up to $20 \%$ [57]. Indeed, one of the potential advantages of MUNE over CMAP is that it is capable of capturing disease progression very early in the disease course, presumably when reinnervation may still be preserving CMAP size but the MUNE is already decreasing [58]. Yet this is offset by the fact that repeatability of MUNE is usually worst early in the disease and improves as the disease reaches end stage and there are fewer, larger SMUPs. Nonetheless, MUNE has been used in several clinical studies and remains a topic of investigation in ALS and in other motor neuron disorders, including spinal muscular atrophy [54].

The limitations to all electrophysiological methods that rely on nerve conduction include the fact that they are limited to nerves that can be effectively stimulated and muscles that can be effectively measured. Most methods have been used only the median and ulnar nerves, but there is no reason that peroneal or tibial motor conduction studies could not also be performed and evaluated. However, superimposed compression neuropathies, common in ALS, could also affect the accuracy of these measures.

\section{Electrical Impedance Myography}

In electrical impedance myography (EIM), a minute electrical current is applied across electrodes placed over a muscle or muscle group of interest and the consequent surface voltages measured [59]. These surface voltages reflect the conductivity of the underlying tissue, as well as the size, number, and health 
of the underlying muscle fibers. Thus, the method is not truly electrophysiological, in the sense that it is not producing excitation of muscle fibers, but is rather electromorphological. Studies of EIM to date have shown substantial sensitivity to ALS disease status and rate of progression, and high reliability [60-62]. Animal work has also strongly supported this concept $[63,64]$. Another advantage of the technique is that it is easy to perform quickly and requires minimal training. And, unlike nerve conduction studies, it can be readily applied to most superficial muscles, including proximal and even bulbar muscles (e.g., the tongue is currently being studied). A recent study has also shown that it correlates to strength testing in patients with ALS [65]. One simple way of conceiving EIM is that it provides data that can be considered a surrogate for CMAP, but, unlike CMAP, does not require nerve stimulation to obtain, and thus can be performed on any sufficiently large superficial muscle.

Thus, while EIM remains promising, it has only been used to a limited extent and has been mostly studied by just a single laboratory (under my direction). Therefore, additional research incorporating the technology into clinical trials is needed to confirm its long-term value in tracking ALS progression.

\section{Peripheral Nerve Excitability Testing}

Excitability testing of peripheral nerves has recently garnered increased attention as studies have demonstrated alterations of excitability of motor neurons in ALS [66, 67]. In addition, 1 study showed that the degree of excitability at baseline predicted long-term survival in humans, with higher levels of excitability predicting shorter survival [68]. Whereas these data appear promising, the value of excitability metrics for serving as markers of disease progression still requires further study.

\section{Transcranial Magnetic Stimulation Measures}

Nearly all the measures discussed in this review have focused on the lower motor neuron in the anterior horn of the spinal cord. And, unfortunately, methods for assessing upper motor neuron dysfunction in the disease are decidedly limited. However, in the early 1990s, several studies showed that cortical excitability was altered in ALS by the use of transcranial magnetic stimulation (TMS) [69-71]. Many studies since then have been pursued, and a number of measures appear to be altered in ALS, including motor threshold (generally reduced early in the disease, supporting the presence of increased excitability), motor-evoked potential (generally also increased early in the disease), central motor conduction time (generally prolonged in the disease), and cortical silent period (reduced in the disease) [72]. While such differences in ALS are identifiable, how well each tracks disease progression is less well studied. Whereas 1 investigation has suggested that TMS could serve as a biomarker to evaluate disease progression [73], another failed to show a clear longitudinal change in TMS parameters [74].

Peripheral Nerve and Muscle Imaging with Ultrasound and MRI

As motor neuron loss and muscle atrophy are primary features of ALS, it is not surprising that radiological techniques will identify reductions in nerve and muscle size. In the case of ultrasound, cross-sectional changes are readily observable [75]. Moreover, ultrasound studies have demonstrated the presence of fasciculations [76, 77], and even fibrillations $[78,79]$. And while it has the potential to serve as useful measure to assist with disease diagnosis, its value as a biomarker of disease progression is less certain. One study suggested that it was not sufficiently sensitive to capture disease progression, at least by measuring muscle thickness [80]. Further work in this field, however, is clearly needed.

Most MRI studies in ALS have focused on imaging the brain and spinal cord and not muscle, and this topic is discussed in a separate article included in this issue. Given the cost and other challenges of using MRI (including the fact that patients generally must remain supine during a study, a posture that becomes increasingly challenging with progressive disease) it has not been used routinely to assess muscle condition. Nonetheless, studies have shown increased T2 signal in muscle [81], and 1 study has shown progressive loss of muscle volume in ALS mice [82]. However, like ultrasound, it sensitivity to progression in the human disease again appears dubious in that subclinical disease progression was not identifiable [83].

\section{Serum and Cerebrospinal Fluid Biomarkers}

The search for "wet" biomarkers continues to be a major effort by a number of laboratories. A number of easily measured and obtainable tests such as creatine kinase [84], uric acid [85], and serum cholesterol [86] have been studied, but none have been identified as effective biomarkers of disease progression. Some, however, such as uric acid [85], and triglyceride and cholesterol levels [86, 87], do correlate to survival.

In addition to these readily obtained laboratory tests, a variety of disease-specific biomarkers have been sought including neurofilament heavy subunit, both in serum and cerebrospinal fluid (CSF) $[88,89]$, and CSF cystatin C [90, 91]. Cystatin $\mathrm{C}$ levels have also been found to correlate with the rate of disease progression and are predictive of survival [90]. While these proteins are increased in ALS patients, neither has yet been shown to be better than other established markers, such as the ALSFRS-R or electrophysiological measures for following disease progression. Still, much research is ongoing 
in the hope of identifying an easily obtainable biomarker from serum or CSF that can be used to quantify disease progression and the impact of an effective therapy.

\section{Survival}

Strictly speaking, of course, the length of survival from the time of symptom onset cannot be considered a clinical marker of disease progression. Yet the 1 drug that has been approved for use to slow the progression of ALS, riluzole, was proven not with any of the approaches discussed above, but rather survival [92]. Moreover, the recent trial of dexpramipexole in ALS utilized a rank combination of ALSFRS-R and survival as the trial's outcome measures [10]. However, there are problems using survival as an outcome measure. First, it requires that the study be sufficiently long and large enough to have a substantial number of subjects die or require full ventilation during the study period. Second, the timing of death/full ventilatory support may be affected by a variety of factors ranging from the use of supportive therapies, including bilevel positive airway pressure and gastrostomy tubes, to the patient's socioeconomic status [93]. Thus, survival does not represent a true gold standard as it also can be influenced by a variety of outside factors.

\section{Conclusions: Taking Stock and Future Directions}

So where are we when it comes to monitoring disease progression in ALS clinical trials? It appears that while there is a plethora of potential tools, there continues to be debate as to which is most promising. Clinical measures that are closely tied to patient health status, including the ALSFRS-R and handheld dynamometry, are certainly useful. However, in my opinion, neurophysiologic measures may offer the best compromise, capturing an outcome closely related to function but that is effort independent. But given ALS's variable involvement of the central versus peripheral nervous system and the limb most affected, applying these in a consistent fashion still offers unique challenges.

The US FDA has defined a program of biomarker qualification, in which biomarkers can be developed as tools to assist in drug testing and, ultimately, drug approval [94]. The idea is that such measures, which are defined as "pharmacodynamic biomarkers" and cannot be influenced by patient effort or mood, can make clinical trials more efficient than by employing standard measures. And if one of these markers is proven out sufficiently, it can achieve the designation of being a "surrogate marker", in which it can be used in lieu of standard clinical outcomes in a phase 3 clinical trial to gain regulatory approval. While all of the nonclinical biomarkers discussed here are far from reaching surrogate status, it is worth considering pursuit of this designation as a worthwhile effort.

Another challenge to consider is the application of localized biomarkers depending on the therapy offered. For example, in studies of stem cell transplantation, utilizing wholebody measures of disease progression may problematical. If injection of stem cells into the cervical spine is employed specifically with the goal of improving pulmonary function, measuring the FVC is probably more important than evaluating ALSFRS-R or strength. Parallel arguments can be made for the lumbar injection of cells, where directed measurements of the lower limbs are needed. Indeed, one completed stem cell study relied on a combination of localized functional and physiological measures to achieve these goals [95].

Finally, just as we focus on identifying new therapies to halt or reverse the progress of the disease, it is also important for us to consider these studies as opportunities to test and improve biomarkers on an ongoing basis. As noted, the ALSFRS-R, while useful, could likely be improved to yield a tool with improved sensitivity and greater reliability. Similarly, individual or combinations of electrophysiological markers may offer greater value than the ones tested to date. It is only through the concurrent testing of biomarkers along with therapies that we can hope to identify an optimized set of tools for the evaluation of ALS progression and, ultimately, the effect of therapy.

Required Author Forms Disclosure forms provided by the authors are available with the online version of this article.

\section{References}

1. Norris FH, Calanchini PR, Fallat RJ, Panchari S, Jewett B. The administration of guanidine in amyotrophic lateral sclerosis. Neurology 1974;24:721-728.

2. Appel V, Stewart SS, Smith G, Appel SH. A rating scale for amyotrophic lateral sclerosis: description and preliminary experience. Ann Neurol 1987;22:328-333.

3. Cedarbaum JM, Stambler N. Performance of the Amyotrophic Lateral Sclerosis Functional Rating Scale (ALSFRS) in multicenter clinical trials. J Neurol Sci 1997;152(Suppl.):S1-S9.

4. Cedarbaum JM, Stambler N, Malta E, et al. The ALSFRS-R: a revised ALS functional rating scale that incorporates assessments of respiratory function. BDNF ALS Study Group (Phase III). J Neurol Sci 1999;169:13-21.

5. Kaufmann P, Levy G, Montes J, et al. Excellent inter-rater, intra-rater, and telephone-administered reliability of the ALSFRS-R in a multicenter clinical trial. Amyotroph Lateral Scler 2007;8:42-46.

6. Gordon PH, Miller RG, Moore DH. ALSFRS-R. Amyotroph Lateral Scler Other Motor Neuron Disord 2004;5(Suppl. 1):90-93.

7. Miano B, Stoddard G, Davis S, Bromberg M. Inter-evaluator reliability of the ALS Functional Rating Scale. Amyotroph Lateral Scler 2004;5:235-239. 
8. Kimura F, Fujimura C, Ishida S, et al. Progression rate of ALSFRS-R at time of diagnosis predicts survival time in ALS. Neurology 2006;66:265-27.

9. Shefner JM, Cudkowicz ME, Schoenfeld D, et al. A clinical trial of creatine in ALS. Neurology 2004;63:1656-1661.

10. Cudkowicz ME, van den Berg LH, Shefner JM, et al. Dexpramipexole versus placebo for patients with amyotrophic lateral sclerosis (EMPOWER): a randomised, double-blind, phase 3 trial. Lancet Neurol 2013;12:1059-1067.

11. Jansen LA. Two concepts of therapeutic optimism. J Med Ethics 2011;37:563-566.

12. Rutkove SB, Caress JB, Cartwright MS, et al. Electrical impedance myography as a biomarker to assess ALS progression. Amyotroph Lateral Scler 2012;13:439-445.

13. Ilse B, Prell T, Walther M, et al. Relationships between disease severity, social support and health-related quality of life in patients with amyotrophic lateral sclerosis. Soc Indic Res 2014. doi:10.1007/ s11205-014-0621-y.

14. Franchignoni F, Mora G, Giordano A, Volanti P, Chiò A. Evidence of multidimensionality in the ALSFRS-R Scale: a critical appraisal on its measurement properties using Rasch analysis. J Neurol Neurosurg Psychiatry 2013;84:1340-1345.

15. Wicks P, Vaughan TE, Massagli MP, Heywood J. Accelerated clinical discovery using self-reported patient data collected online and a patient-matching algorithm. Nat Biotechnol 2011;29:411-414.

16. Burns TM, Graham CD, Rose MR, Simmons Z. Quality of life and measures of quality of life in patients with neuromuscular disorders. Muscle Nerve 2012;46:9-25.

17. Clarke S, Hickey A, O’Boyle C, Hardiman O. Assessing individual quality of life in amyotrophic lateral sclerosis. Qual Life Res 2001;10:149-158.

18. Wright W. Muscle training in the treatment of infantile paralysis. J Bost Med Surg 1912;167:567.

19. Medical Research Council of the United Kingdom. Aids to examination of the peripheral nervous system, Memorandum No. 45. Palo Alto, CA, Pedragon House, 1978.

20. Wadsworth CT, Krishnan R, Sear M, Harrold J, Nielsen DH. Intrarater reliability of manual muscle testing and hand-held dynametric muscle testing. Phys Ther 1987;67:1342-1347.

21. Cuthbert SC, Goodheart GJ. On the reliability and validity of manual muscle testing: a literature review. Chiropr Osteopat 2007;15:4

22. Gordon PH, Moore DH, Miller RG, et al. Efficacy of minocycline in patients with amyotrophic lateral sclerosis: a phase III randomised trial. Lancet Neurol 2007;6:1045-1053.

23. Mulroy SJ, Lassen KD, Chambers SH, Perry J. The ability of male and female clinicians to effectively test knee extension strength using manual muscle testing. J Orthop Sports Phys Ther 1997;26:192-199.

24. Andres PL, Hedlund W, Finison L, Conlon T, Felmus M, Munsat TL. Quantitative motor assessment in amyotrophic lateral sclerosis. Neurology 1986;36:937-937.

25. Andres PL, Finison LJ, Conlon T, Thibodeau LM, Munsat TL. Use of composite scores (megascores) to measure deficit in amyotrophic lateral sclerosis. Neurology 1988;38:405-408.

26. ALS CNTF Treatment Study Group. A double-blind placebo-controlled clinical trial of subcutaneous recombinant human ciliary neurotrophic factor (rHCNTF) in amyotrophic lateral sclerosis. Neurology 1996;46:1244-1244.

27. Cudkowicz ME, Shefner JM, Schoenfeld DA, et al. A randomized, placebo-controlled trial of topiramate in amyotrophic lateral sclerosis. Neurology 2003;61:456-464.

28. Miller RG, Moore DH, Gelinas DF, et al. Phase III randomized trial of gabapentin in patients with amyotrophic lateral sclerosis. Neurology 2001;56:843-848.

29. Group GLALSS. A comparison of muscle strength testing techniques in amyotrophic lateral sclerosis. Neurology 2003;61:1503-1507.
30. Andres PL, Skerry LM, Munsat TL, et al. Validation of a new strength measurement device for amyotrophic lateral sclerosis clinical trials. Muscle Nerve 2012;45:81-85.

31. Andres PL, English R, Mendoza M, et al. Developing normalized strength scores for neuromuscular research. Muscle Nerve 2013;47: 177-182.

32. Beck M, Giess R, Würffel W, Magnus T, Ochs G, Toyka KV. Comparison of maximal voluntary isometric contraction and Drachman's hand-held dynamometry in evaluating patients with amyotrophic lateral sclerosis. Muscle Nerve 1999;22:1265-1270.

33. Pearn J. Two early dynamometers. An historical account of the earliest measurements to study human muscular strength. J Neurol Sci 1978;37:127-134.

34. Bohannon RW. Intertester reliability of hand-held dynamometry: a concise summary of published research. Percept Mot Skills 1999;88: 899-902.

35. Goonetilleke A, Modarres-Sadeghi H, Guiloff RJ. Accuracy, reproducibility, and variability of hand-held dynamometry in motor neuron disease. J Neurol Neurosurg Psychiatry 1994;57:326-332.

36. Schiffman PL, Belsh JM. Pulmonary function at diagnosis of amyotrophic lateral sclerosis; Rate of deterioration. Chest 1993;103:508513.

37. Stambler N, Charatan M, Cedarbaum JM. Prognostic indicators of survival in ALS. ALS CNTF Treatment Study Group. Neurology 1998;50:66-72.

38. Pastula DM, Moore DH, Bedlack RS. Creatine for amyotrophic lateral sclerosis/motor neuron disease. Cochrane Database Syst Rev 2010:CD005225.

39. Schmidt EP, Drachman DB, Wiener CM, Clawson L, Kimball R, Lechtzin N. Pulmonary predictors of survival in amyotrophic lateral sclerosis: use in clinical trial design. Muscle Nerve 2006;33:127-132.

40. Yunusova Y, Green JR, Wang J, Pattee G, Zinman L. A protocol for comprehensive assessment of bulbar dysfunction in amyotrophic lateral sclerosis (ALS). J Vis Exp 2011;Feb 21;(48).

41. Ball LJ, Willis A, Beukelman DR, Pattee GL. A protocol for identification of early bulbar signs in amyotrophic lateral sclerosis. J Neurol Sci 2001;191:43-53.

42. Clark HM, Solomon NP. Age and sex differences in orofacial strength. Dysphagia 2012;27:2-9.

43. Wallace KL, Middleton S, Cook IJ. Development and validation of a self-report symptom inventory to assess the severity of oralpharyngeal dysphagia. Gastroenterology 2000;118:678-687.

44. Farkas C, Hamilton-Wright A, Parsaei H, Stashuk DW. A Review of clinical quantitative electromyography. Crit Rev Biomed Eng 2010;38:467-485.

45. Kimura J. Electrodiagnosis in diseases of nerve and muscle: principles and practice. Third edition. Oxford University Press, New York, 2001.

46. Shefner JM, Watson ML, Simionescu L, et al. Multipoint incremental motor unit number estimation as an outcome measure in ALS. Neurology 2011;77:235-241.

47. Neuwirth C, Nandedkar S, Stalberg E, Weber M. Motor unit number index (MUNIX): a novel neurophysiological technique to follow disease progression in amyotrophic lateral sclerosis. Muscle Nerve 2010;42:379-384.

48. Lewelt A, Krosschell KJ, Scott C, et al. Compound muscle action potential and motor function in children with spinal muscular atrophy. Muscle Nerve 2010;42:703-708.

49. Neuwirth C, Nandedkar S, Stalberg E, et al. Motor Unit Number Index (MUNIX): reference values of five different muscles in healthy subjects from a multi-centre study. Clin Neurophysiol 2011;122: 1895-1898.

50. Swash M, de Carvalho M. The Neurophysiological index in ALS. Amyotroph Lateral Scler Other Mot Neuron Disord 2004;5(Suppl. 1):108-110. 
51. Cheah BC, Vucic S, Krishnan A V, Boland RA, Kiernan MC. Neurophysiological index as a biomarker for ALS progression: validity of mixed effects models. Amyotroph Lateral Scler 2011;12:33-38.

52. McComas AJ, Fawcett PR, Campbell MJ, Sica RE. Electrophysiological estimation of the number of motor units within a human muscle. J Neurol Neurosurg Psychiatry 1971;34:121-131.

53. Doherty TJ, Brown WF. The estimated numbers and relative sizes of thenar motor units as selected by multiple point stimulation in young and older adults. Muscle Nerve 1993;16:355-366.

54. Bromberg MB. Updating motor unit number estimation (MUNE). Clin Neurophysiol 2007;118:1-8.

55. Nandedkar SD, Nandedkar DS, Barkhaus PE, Stalberg EV. Motor unit number index (MUNIX). IEEE Trans Biomed Eng 2004;51: 2209-2211.

56. Shefner JM. Motor unit number estimation in human neurological diseases and animal models. Clin Neurophys 2001;112: 955-964.

57. Shefner JM, Cudkowicz ME, Zhang H, Schoenfeld D, Jillapalli D. The use of statistical MUNE in a multicenter clinical trial. Muscle Nerve 2004;30:463-469.

58. Felice KJ. A longitudinal study comparing thenar motor unit number estimates to other quantitative tests in patients with amyotrophic lateral sclerosis. Muscle Nerve 1997;20:179-185.

59. Rutkove SB. Electrical impedance myography: Background, current state, and future directions. Muscle Nerve 2009;40:936-946.

60. Rutkove SB, Caress JB, Cartwright MS, et al. Electrical impedance myography as a biomarker to assess ALS progression. Amyotroph Lateral Scler 2012;13:439-345.

61. Rutkove SB, Zhang H, Schoenfeld DA, et al. Electrical impedance myography to assess outcome in amyotrophic lateral sclerosis clinical trials. Clin Neurophysiol 2007;118:2413-2418.

62. Rutkove SB, Lee KS, Shiffman CA, Aaron R. Test-retest reproducibility of $50 \mathrm{kHz}$ linear-electrical impedance myography. Clin Neurophys 2006;117:1244-1248.

63. Li J, Sung M, Rutkove SB. Electrophysiologic biomarkers for assessing disease progression and the effect of riluzole in SOD1 G93A ALS mice. PLoS ONE 2013;8:e65976.

64. Wang LL, Spieker AJ, Li J, Rutkove SB. Electrical impedance myography for monitoring motor neuron loss in the SOD1 G93A amyotrophic lateral sclerosis rat. Clin Neurophysiol 2011;122:25052511.

65. Rutkove SB, Caress JB, Cartwright MS, et al. Electrical impedance myography correlates with standard measures of ALS severity. Muscle Nerve 2014;49:441-443.

66. Kanai K, Kuwabara S, Misawa S, et al. Altered axonal excitability properties in amyotrophic lateral sclerosis: Impaired potassium channel function related to disease stage. Brain 2006;129:953-962.

67. Pambo-Pambo A, Durand J, Gueritaud JP. Early excitability changes in lumbar motoneurons of transgenic SOD1G85R and SOD1G(93ALow) mice. J Neurophysiol 2009;102:3627-3642.

68. Kanai K, Shibuya K, Kuwabara S. [Motor axonal excitability properties are strong predictors for survival in amyotrophic lateral sclerosis]. Rinsho Shinkeigaku 2011;51:1118-1119 (in Japanese).

69. Caramia MD, Cicinelli P, Paradiso C, et al. 'Excitability changes of muscular responses to magnetic brain stimulation in patients with central motor disorders. Electroencephalogr Clin Neurophysiol 1991;81:243-250.

70. Prout AJ, Eisen AA. The cortical silent period and amyotrophic lateral sclerosis. Muscle Nerve 1994;17:217-223.

71. Claus D, Brunhölzl C, Kerling FP, Henschel S. Transcranial magnetic stimulation as a diagnostic and prognostic test in amyotrophic lateral sclerosis. J Neurol Sci 1995;129:30-34.
72. Vucic S, Ziemann U, Eisen a, Hallett M, Kiernan MC. Transcranial magnetic stimulation and amyotrophic lateral sclerosis: Pathophysiological insights. J Neurol Neurosurg Psychiatry 2013;84:1161-1170.

73. Floyd AG, Yu QP, Piboolnurak P, et al. Transcranial magnetic stimulation in ALS: Utility of central motor conduction tests. Neurology 2009; 72:498-504

74. Mills KR. The natural history of central motor abnormalities in amyotrophic lateral sclerosis. Brain 2003;126:2558-2566.

75. Cartwright MS, Walker FO, Griffin LP, Caress JB. Peripheral nerve and muscle ultrasound in amyotrophic lateral sclerosis. Muscle Nerve 2011;44:346-351

76. Pillen S, van Alfen N. Skeletal muscle ultrasound. Neurol Res 2011;33:1016-1024.

77. Pillen S, Arts IM, Zwarts MJ. Muscle ultrasound in neuromuscular disorders. Muscle Nerve 2008;37:679-693.

78. Van Alfen N, Nienhuis M, Zwarts MJ, Pillen S. Detection of fibrillations using muscle ultrasound: diagnostic accuracy and identification of pitfalls. Muscle Nerve 2011;43:178-182.

79. Pillen S, Nienhuis M, van Dijk JP, Arts IM, van Alfen N, Zwarts MJ. Muscles alive: ultrasound detects fibrillations. Clin Neurophysiol 2009;120:932-936.

80. Arts IMP, Overeem S, Pillen S, Jurgen Schelhaas H, Zwarts MJ. Muscle changes in amyotrophic lateral sclerosis: A longitudinal ultrasonography study. Clin Neurophysiol 2011;122: 623-628.

81. Goodin DS, Rowley HA, Olney RK. Magnetic resonance imaging in amyotrophic lateral sclerosis. Ann Neurol 1988;23:418-420.

82. Brooks KJ, Hill MDW, Hockings PD, Reid DG. MRI detects early hindlimb muscle atrophy in Gly93Ala superoxide dismutase-1 (G93A SOD1) transgenic mice, an animal model of familial amyotrophic lateral sclerosis. NMR Biomed 2004; 17:28-32.

83. Jenkins TM, Burness C, Connolly DJ, et al. A prospective pilot study measuring muscle volumetric change in amyotrophic lateral sclerosis. Amyotroph Lateral Scler Frontotemporal Degener 2013;14:414423.

84. Iłzecka J, Stelmasiak Z. Creatine kinase activity in amyotrophic lateral sclerosis patients. Neurol Sci 2003;24:286-287.

85. Paganoni S, Zhang M, Zárate AQ, et al. Uric acid levels predict survival in men with amyotrophic lateral sclerosis. J Neurol 2012;259:1923-1928.

86. Dorst J, Kühnlein P, Hendrich C, Kassubek J, Sperfeld AD, Ludolph AC. Patients with elevated triglyceride and cholesterol serum levels have a prolonged survival in amyotrophic lateral sclerosis. J Neurol 2011;258:613-617.

87. Chiò A, Calvo A, Ilardi A, et al. Lower serum lipid levels are related to respiratory impairment in patients with ALS. Neurology 2009;73: $1681-1685$

88. Mendonça DMF, Martins SCS, Higashi R, et al. Neurofilament heavy subunit in cerebrospinal fluid: a biomarker of amyotrophic lateral sclerosis? Amyotroph Lateral Scler 2011;12:144-147.

89. Boylan KB, Glass JD, Crook JE, et al. Phosphorylated neurofilament heavy subunit (pNF-H) in peripheral blood and CSF as a potential prognostic biomarker in amyotrophic lateral sclerosis. J Neurol Neurosurg Psychiatry 2013;84:467-472.

90. Wilson ME, Boumaza I, Lacomis D, Bowser R. Cystatin C: A candidate biomarker for amyotrophic lateral sclerosis. PLoS ONE 2010;5:e15133.

91. Tsuji-Akimoto S, Yabe I, Niino M, Kikuchi S, Sasaki H. Cystatin C in cerebrospinal fluid as a biomarker of ALS. Neurosci Lett 2009;452:52-55. 
92. Bensimon G, Lacomblez L, Meininger V. A controlled trial of riluzole in amyotrophic lateral sclerosis. ALS/Riluzole Study Group. N Engl J Med 1994;330:585-591.

93. Paillisse C, Lacomblez L, Dib M, Bensimon G, Garcia-Acosta S, Meininger V. Prognostic factors for survival in amyotrophic lateral sclerosis patients treated with riluzole. Amyotroph Lateral Scler Other Motor Neuron Disord 2005;6:37-44.
94. Biomarker Qualification Context of Use n.d. http://www.fda.gov/ D r u g s / D e v e lo p m e n t A p r o va $1 \mathrm{Proces} \mathrm{s/}$ DrugDevelopmentToolsQualificationProgram/ucm284620.htm. Accessed 2 Jan 2015.

95. Feldman EL, Boulis NM, Hur J, et al. Intraspinal neural stem cell transplantation in amyotrophic lateral sclerosis: phase 1 trial outcomes. Ann Neurol 2014;75:363-373. 